\title{
Preparation and Characterization of K-Carrageenan/Nanosilica Biocomposite Film
}

\author{
Lokesh R. Rane, Niranjan R. Savadekar, Pravin G. Kadam, and Shashank T. Mhaske \\ Department of Polymer and Surface Engineering, Institute of Chemical Technology, Matunga (E), Mumbai, \\ Maharashtra 400 019, India \\ Correspondence should be addressed to Shashank T. Mhaske; stmhaske@gmail.com
}

Received 8 November 2013; Revised 12 January 2014; Accepted 20 January 2014; Published 3 March 2014

Academic Editor: Peter Chang

Copyright (C) 2014 Lokesh R. Rane et al. This is an open access article distributed under the Creative Commons Attribution License, which permits unrestricted use, distribution, and reproduction in any medium, provided the original work is properly cited.

\begin{abstract}
The purpose of this study is to improve the performance properties of K-carrageenan (K-CRG) by utilizing nanosilica (NSI) as the reinforcing agent. The composite films were prepared by solution casting method. NSI was added up to $1.5 \%$ in the K-CRG matrix. The prepared films were characterized for mechanical (tensile strength, tensile modulus, and elongation at break), thermal (differential scanning calorimetry, thermogravimetric analysis), barrier (water vapour transmission rate), morphological (scanning electron microscopy), contact angle, and crystallinity properties. Tensile strength, tensile modulus, and crystallinity were found to have increased by $13.8,15$, and $48 \%$ whereas water vapour transmission rate was found to have decreased by $48 \%$ for $0.5 \%$ NSI loaded K-CRG composite films. NSI was found to have formed aggregates for concentrations above $0.5 \%$ as confirmed by scanning electron microscopy. Melting temperature, enthalpy of melting, and degradation temperature of K-CRG increased with increase in concentration of NSI in K-CRG. Contact angle also increased with increase in concentration of NSI in K-CRG, indicating the decrease in hydrophilicity of the films improving its water resistance properties. This knowledge of the composite film could make beneficial contributions to the food and pharmaceutical packaging applications.
\end{abstract}

\section{Introduction}

Biopolymers are the polymeric materials obtained from renewable agriculture by-products, waste of food processing industry, and other natural resources (animals, plants, and algae). They are attracting considerable attention as potential replacement for petroleum based plastics due to the increased consciousness for sustainable development. Biopolymers maintain the carbon dioxide balance after their degradation and are readily biodegradable, which will save energy on waste disposal. However, limited performance and high cost of these materials are restricting their competitiveness to traditional thermoplastics. One way to enhance the material properties and to broaden the possible applications for biopolymers is to produce nanocomposites using it [1].

$\mathrm{K}$-carrageenan $(\mathrm{K}-\mathrm{CRG})$ is a generic name for a family of polysaccharides, obtained by extraction from certain species of red seaweeds. K-CRG is a water-soluble biopolymer (at elevated temperature) with a linear chain of partially sulphated galactans, which presents high potential as a gel-forming material [2]. Structure of K-CRG is shown in Figure 1.

Nanosilica (NSI) filled polymer matrix composites have received considerable attention in the past few years. It has been reported that NSI filled polymer matrix composites show a significant improvement in mechanical and thermal properties [3-5]. Studies on NSI dispersion in polymer matrices like poly (methyl methacrylate) [6,7], high density polyethylene [8], and poly(ethylene oxide) [9] have been carried out. However, with the polymer matrices mentioned being hydrophobic in nature and NSI being hydrophilic, various types of surfactants or compatibilizers have been utilized to bring about compatibility between the two noninteracting phases [6-9]. Some attention has also been addressed to nanocomposites based on silica and polymers like poly(vinyl alcohol) [10, 11], poly(vinyl pyrrolidone), and chitosan [10]. As both the polymers used and NSI were hydrophilic in nature, no use of surfactants or compatibilizers was thought of, as both phases were interacting. NSI particles are largely 


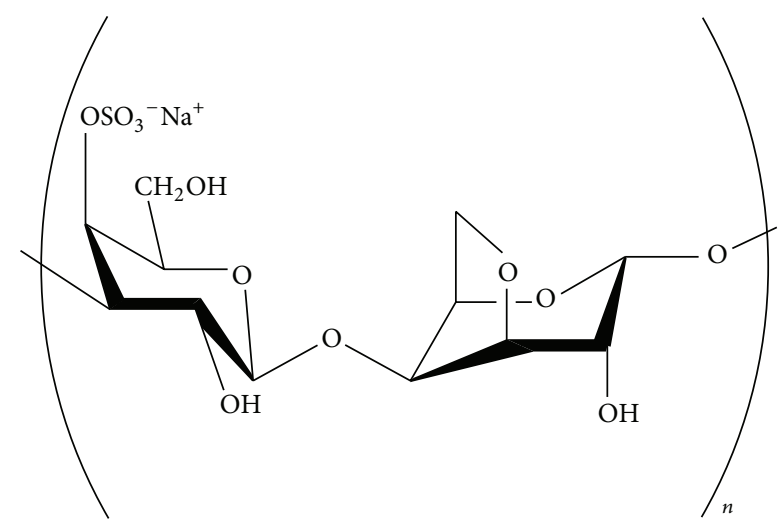

FIGURE 1: Chemical structure of K-carrageenan.

prepared by reverse microemulsion, flame synthesis, chemical vapor deposition, and sol-gel methods [3-5].

The goal of this work is to study the effect of the concentration of NSI on the mechanical, thermal, morphological, barrier, and contact angle properties of K-CRG. K-CRG was used as matrix with glycerol as the plasticizer. K-CRG was added $0.5,1$ and $1.5 \mathrm{wt} \%$ of NSI as the reinforcing agent.

\section{Materials and Experimental Methods}

2.1. Materials. K-carrageenan (K-CRG, 22048, water content: $8.9 \%$, residue on ignition: $25.2 \%$ ) was procured from Fluka Chemie GmbH, Switzerland. Nanosilica (NSI) having average particle size of $50 \pm 10 \mathrm{~nm}$ was obtained from NaBond, China. Glycerol having maximum assay 99.5 (AR grade) was obtained from S. D. Fine-Chem Ltd., Mumbai, India. Distilled water was obtained from Bio Lab Diagnostics India Pvt. Ltd. (Mumbai, India). All materials were used as obtained without any modification.

2.2. Preparation of K-CRG/NSI Composite Films. The required amount of NSI $(0.5,1$, or $1.5 \% \mathrm{w} / \mathrm{w}$ of $\mathrm{K}-\mathrm{CRG})$ was dispersed in $50 \mathrm{~mL}$ of distilled water under continuous stirring at $1500 \mathrm{rpm}$ for $15 \mathrm{~min}$. This dispersion was sonicated in a sonicator bath (SUPERSONICS, India) for $10 \mathrm{~min}$. The solution of K-CRG was prepared separately by adding $2 \mathrm{gm}$ K-CRG biopolymer in $200 \mathrm{~mL}$ distilled water for 1 hour under continuous stirring. The NSI distilled water dispersion was then added slowly in the prepared K-CRG solution, to get $0.5,1$, or $1.5 \mathrm{wt} \%$ MNSI content solutions. Glycerol was added ( $40 \% \mathrm{w} / \mathrm{w}$ of $\mathrm{K}-\mathrm{CRG}$ ) as a plasticizer. The solution was kept under continuous agitation (1500 rpm) for two hours. Then, the temperature of the solution was raised up to $65^{\circ} \mathrm{C}$ and maintained for $10 \mathrm{~min}$ under agitation $(1500 \mathrm{rpm})$ to get a better and uniformly mixed solution. The solution was then cooled down to $45^{\circ} \mathrm{C}$ under continuous agitation. The obtained solutions were cast on the acrylic sheet trays having dimensions of $22 \mathrm{~cm} \times 18 \mathrm{~cm} \times 1 \mathrm{~cm}$. The trays were kept in a hot air circulating oven (Siena Instruments, Mumbai) for drying at $45^{\circ} \mathrm{C}$. Control films or base polymer films (K-CRG) were also prepared by using
TABLE 1: Composition of the prepared K-CRG/NSI composite films with nomenclature.

\begin{tabular}{lcccc}
\hline Sr. no. & Nomenclature & $\begin{array}{c}\text { K-CRG } \\
(\mathrm{g})\end{array}$ & $\begin{array}{c}\text { NSI } \\
\text { gm }(\% \mathrm{w} / \mathrm{w})\end{array}$ & $\begin{array}{c}\text { Glycerol } \\
\text { gm }(\% \mathrm{w} / \mathrm{w})\end{array}$ \\
\hline 1. & PK-CRG & 2.0 & $0(0)$ & $0.8(40)$ \\
2. & K-CRGN0.5 & 2.0 & $0.01(0.5)$ & $0.8(40)$ \\
3. & K-CRGN1.0 & 2.0 & $0.02(1.0)$ & $0.8(40)$ \\
4. & K-CRGN1.5 & 2.0 & $0.03(1.5)$ & $0.8(40)$ \\
\hline
\end{tabular}

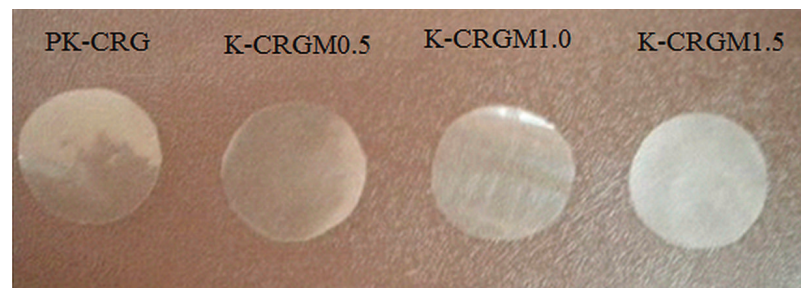

FIGURE 2: Photograph of the prepared PK-CRG and K-CRG/NSI composite films.

the same procedure without addition of NSI for comparative study with the nanocomposite films. Prepared compositions and nomenclature are listed in Table 1 whereas Figure 2 is a photograph of the prepared control and films.

\section{Characterization and Testing}

3.1. Mechanical Properties. In mechanical properties, tensile properties like tensile strength, percent elongation at break, and tensile modulus of the films were determined using a Universal Testing Machine (LR-50K, LLOYD Instruments, UK) with 500N load cell, in accordance with ASTM D 882. Ten samples were tested for each composition.

\subsection{Thermal Properties}

3.2.1. Differential Scanning Calorimeter (DSC). DSC was used to measure thermal transitions of K-CRG films and KCRG/MNSI nanocomposite films. The test was performed with Q100 DSC (TA Instruments) equipment, fitted with a nitrogen-based cooling system. The samples were accurately weighed and sealed in aluminium pans. An empty pan was used as the reference pan. All the measurements were performed in the temperature range from -50 to $150^{\circ} \mathrm{C}$ at a heating rate of $5^{\circ} \mathrm{C} / \mathrm{min}$.

3.2.2. Thermogravimetric Analysis (TGA). TGA analysis was done to determine the effect of MNSI addition on the degradation characteristics of K-CRG film. TGA was done on SDT Q600 (TA Instruments). All the samples were characterized in the temperature range from 40 to $600^{\circ} \mathrm{C}$ at a heating rate $10^{\circ} \mathrm{C} / \mathrm{min}$.

3.3. Rheological Properties. Measurements of solution rheology were made at $30^{\circ} \mathrm{C}$ using a Rheometer (MCR 101, Anton Paar, Graz, Austria) employing the Cone and Plate geometry 
having diameter of $35 \mathrm{~mm}$, measured over a shear rate range from 0.01 to $100 \mathrm{~s}^{-1}$. Frequency sweep measurements for storage modulus $\left(G^{\prime}\right)$, loss modulus $\left(G^{\prime \prime}\right)$, and phase angle $\left(\tan \delta=G^{\prime \prime} / G^{\prime}\right)$ as a function of frequency $\left(\omega, \operatorname{rad~s}^{-1}\right)$ were measured. The frequency of oscillation was varied from 0.01 to $40 \mathrm{rad} / \mathrm{s}$.

3.4. Morphological Analysis. Morphological analysis was done using a scanning electron microscope to investigate the dispersion of MNSI in the K-CRG polymer matrix at various loading levels. The morphology of K-CRG matrix composite films was observed by using a JSM-6380 LA scanning electron microscope (JEOL Co., Japan). Samples were fractured under liquid nitrogen to avoid any disturbance to the molecular structure and then sputtered with platinum before imaging.

3.5. Contact Angle Measurement. Contact angles of the films were measured by the sessile drop method with distilled water using a drop shape instrument Thermo Cahn Radian series 300 instrument.

3.6. Barrier Properties. Water vapor transmission rates (WVTR) values of the films were determined gravimetrically according to ASTM E96 method. Each test film was sealed on the top of permeation cells containing distilled water. The permeation cells were placed in desiccators maintained at $0 \%$ $\mathrm{RH}$. The water transferred through the film and absorbed by the desiccant was determined from the weight of the permeation cell. Anhydrous calcium chloride $\left(\mathrm{CaCl}_{2}\right)$ was used as a desiccant. Each permeation cell was weighed at an interval of $24 \mathrm{hr}$. The WVTR was expressed in $\mathrm{gm} \cdot \mathrm{mm} / \mathrm{cm}^{2}$ per day.

3.7. X-Ray Diffraction Analysis. Wide angle X-ray diffraction analysis was used to determine the crystallinity of the KCRG/MNSI composite films. It was performed on a Miniflex $\mathrm{X}$-ray Diffractometer (Rigaku, Japan) using a $\mathrm{CuK}_{\alpha}$ radiation source having wavelength of $1.54 \AA$ with $\mathrm{Ni}$ filter. The diffractometer was operated at $30 \mathrm{KV}, 15 \mathrm{~mA}$ at a scanning rate of $2^{\circ} / \mathrm{min}$. Angle range was taken from 2 to $40^{\circ}$. Data processing was done using the Jade 6.0 software.

\section{Results and Discussion}

4.1. Mechanical Properties. In mechanical property, tensile test was performed in order to understand the effect of NSI addition of the strength of the prepared K-CRG composite films. Tensile properties like tensile strength (TS), percentage elongation at break (EB), and tensile modulus (TM) were determined and are shown in graphical form in Figures 3, 4, and 5 , respectively.

TS and TM were found to have increased by 13.8 and $15 \%$, respectively, and was the highest improvement obtained, for just $0.5 \%$ addition of NSI in K-CRG. This improvement is appreciable compared to the amount of NSI added. At concentration of NSI above $0.5 \%$, TS and TM both started deteriorating. Thus, $0.5 \%$ is the optimized concentration to

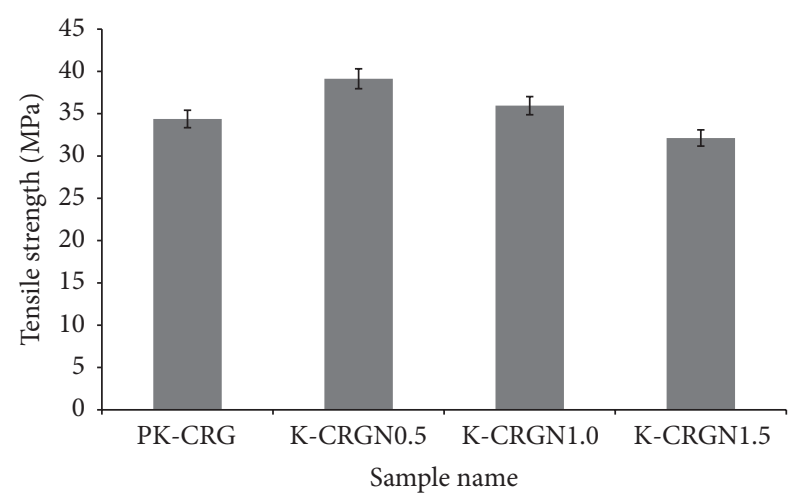

FIGURE 3: Effect of NSI concentration on tensile strength of K-CRG films.

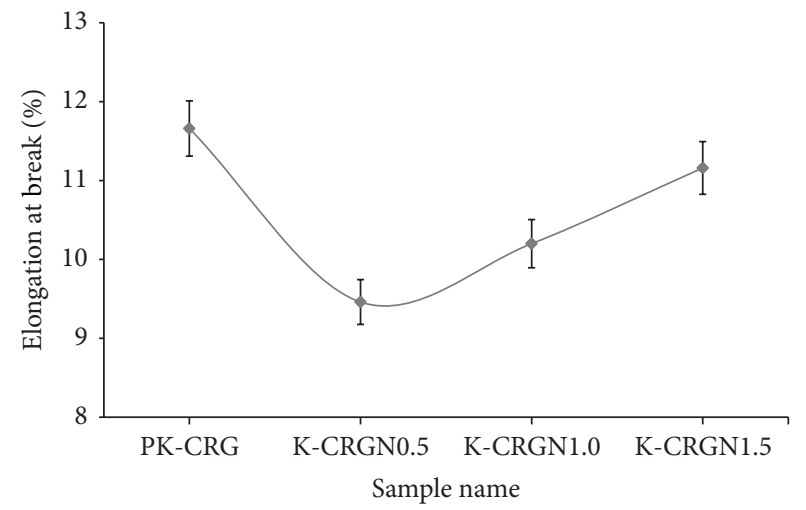

FIGURE 4: Effect of NSI concentration on the elongation property of K-CRG films.

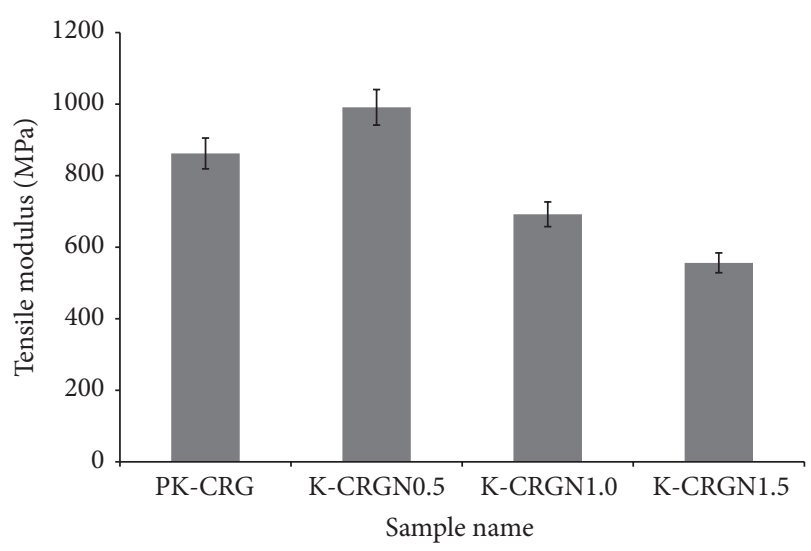

FIGURE 5: Effect of NSI concentration on the tensile modulus of KCRG films.

be added to improve the TS and TM of K-CRG by the highest possible extent. NSI and K-CRG both being hydrophilic in nature had better interaction with each other. This interaction increased the orientation of K-CRG polymeric chains about NSI increasing the crystallinity of K-CRG. At $0.5 \%$ loading, NSI remained uniformly and individually dispersed in the K-CRG matrix while above $0.5 \%$ concentration NSI started forming aggregates, decreasing the effective surface area for 


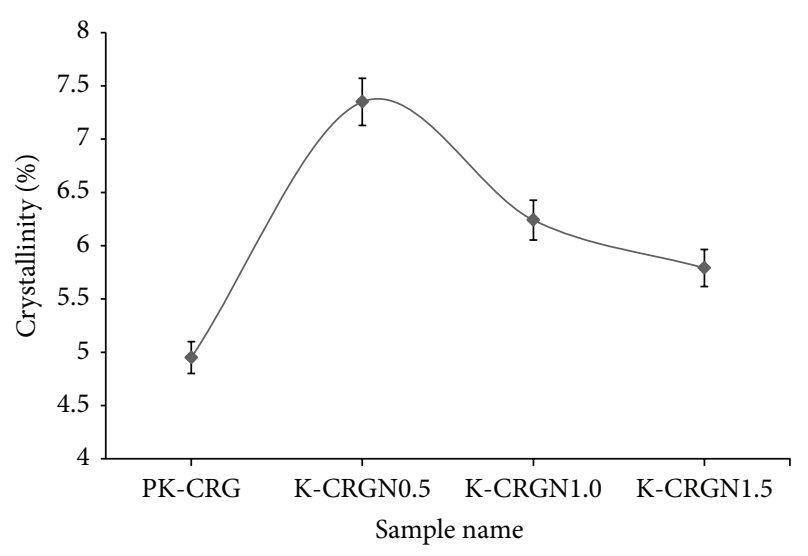

FIGURE 6: Effect of NSI concentration on the crystallinity of K-CRG films.

interacting with K-CRG, decreasing TS and TM. Uniform dispersion at $0.5 \%$ concentration and formation of aggregates above that has been proven by scanning electron microscopy, whereas the increase and decrease in crystallinity have been proven by X-ray diffraction analysis. This increase in crystallinity stiffened the films making them break at lower elongation. Elongation at break decreased for $0.5 \%$ NSI loaded KCRG and, however, increased for concentrations above $0.5 \%$ due to the formation of aggregates and subsequent decrease in crystallinity. NSI has proven effective in improving the mechanical property of K-CRG. Similar observations had been made by Chung et al. [12] for starch/MMT nanobiocomposite films and Savadekar et al. $[13,14]$ for starch and KCRG films reinforced with nanocellulose fibres.

4.2. X-Ray Diffraction Analysis. X-ray diffraction analysis was done to understand the change in crystallinity of K-CRG caused by the addition of NSI. Crystallinity values obtained for the prepared films are shown graphically in Figure 6.

Crystallinity of K-CRG increased on addition of $0.5 \%$ NSI into it and, however, decreased on further addition of NSI. At $0.5 \%$ loading, NSI remained uniformly dispersed in the K-CRG matrix. However, at higher concentration formed aggregates decreasing the interaction with K-CRG, decreasing the crystallinity. This proves the trend observed in the mechanical properties on addition of NSI. Crystallinity of K-CRG was found to have increased by $48 \%$ on $0.5 \%$ addition of NSI. K-CRGN1.0 and K-CRGN1.5 also have higher crystallinity than PK-CRG, but was lesser than KCRGN0.5, due to the formation of aggregates by NSI. Thus, NSI is acting as a nucleating agent in K-CRG. Crystallinity of NSI was found to be $29.57 \%$.

4.3. Thermal Properties. PK-CRG and K-CRG/NSI composite samples were characterized by differential scanning calorimetry (DSC) and thermogravimetric analysis to understand their thermal characteristics.

4.3.1. Differential Scanning Calorimetry (DSC). The calorimetric thermograms obtained for PK-CRG and K-CRG/NSI

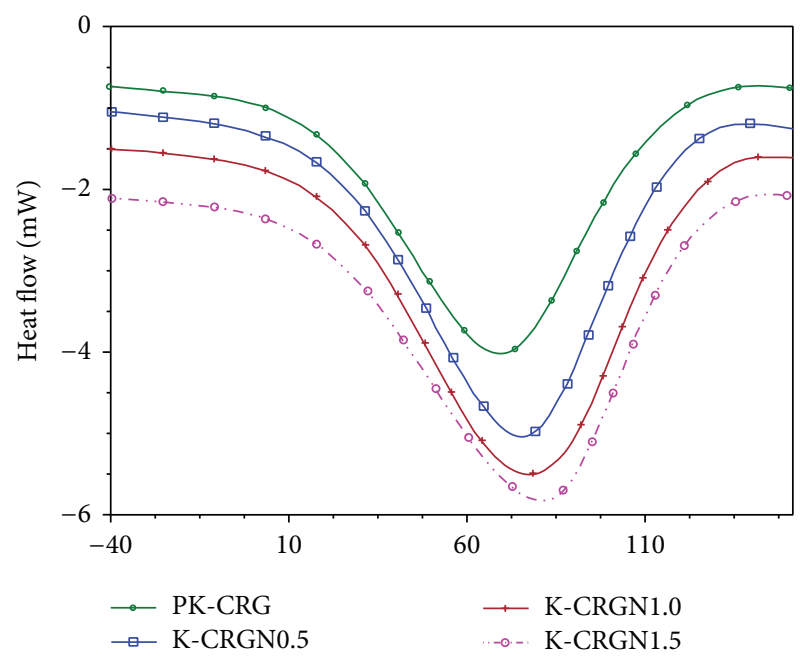

FIGURE 7: Effect of NSI concentration on the DSC thermogram of K-CRG films.

TABLE 2: Effect of NSI concentration on the melting temperature and enthalpy of melting of K-CRG.

\begin{tabular}{lcc}
\hline Sample name & $\begin{array}{c}\text { Melting temperature } \\
\left({ }^{\circ} \mathrm{C}\right)\end{array}$ & $\begin{array}{c}\text { Enthalpy of melting } \\
(\mathrm{J} / \mathrm{g})\end{array}$ \\
\hline PK-CRG & 68.18 & 335.5 \\
K-CRGN0.5 & 74.61 & 363.9 \\
K-CRGN1.0 & 75.74 & 368.6 \\
K-CRGN1.5 & 79.62 & 372.4 \\
\hline
\end{tabular}

composite films are depicted in Figure 7 as a plot of heat flow $(\mathrm{mW})$ versus temperature $\left({ }^{\circ} \mathrm{C}\right)$ whereas the values of melting temperature $\left(T_{m},{ }^{\circ} \mathrm{C}\right)$ and enthalpy of melting $\left(H_{m}, \mathrm{~J} / \mathrm{g}\right)$ are listed in Table 2.

The DSC thermograms of PK-CRG and K-CRG/NSI composite films showed quite broad melting transition temperature range. Melting temperature of PK-CRG was found to be around $68^{\circ} \mathrm{C}$ requiring about $335.5 \mathrm{~J} / \mathrm{g}$ energy to undergo melting. $T_{m}$ and $H_{m}$ increased with increased addition of NSI in K-CRG. NSI and K-CRG are both hydrophilic in nature. Thus, NSI got nicely dispersed in the K-CRG matrix up to $0.5 \%$ concentration and, however, formed aggregates at higher concentration. These aggregates negatively affected the mechanical property and crystallinity but had positive effect on thermal property. Silica is a thermally insulating material. NSI is thus also thermally insulating. In addition, the crystallinity induced by NSI addition (for all compositions) must also have helped in increasing the thermal resistance of K-CRG. As the concentration of NSI increased in K-CRG, its better dispersion, induced crystallinity, and thermal insulating property increased the thermal stability of K-CRG. $T_{m}$ and $H_{m}$ of K-CRG had increased by about 16.8 and $11 \%$ on just $1.5 \%$ addition of NSI.

4.3.2. Thermogravimetric Analysis (TGA). The TGA technique is useful to study complex systems like polymer blends $[15,16]$ and nanocomposites $[17,18]$. The thermal 


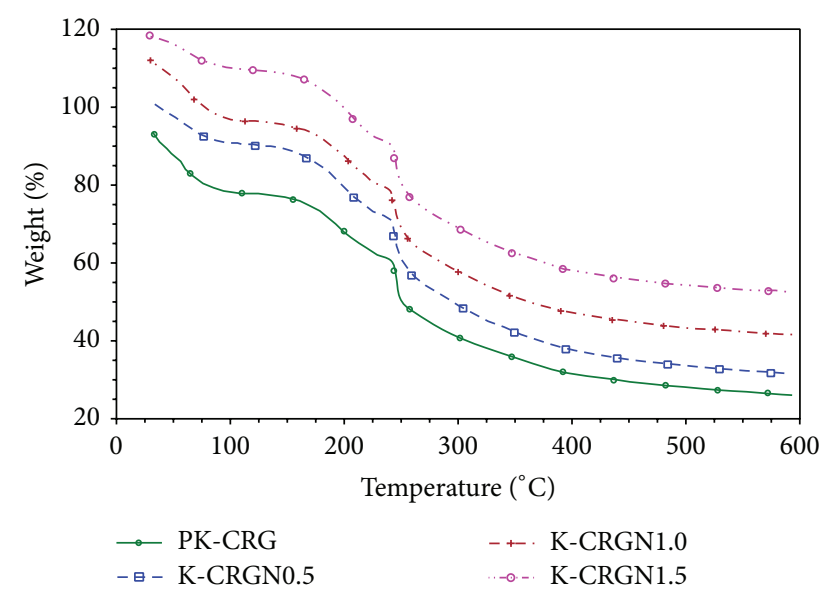

FIGURE 8: Effect of NSI addition on the thermal degradation characteristic of K-CRG films.

TABLE 3: Effect of NSI concentration on the degradation temperature of K-CRG.

\begin{tabular}{lcc}
\hline \multirow{2}{*}{ Sample name } & \multicolumn{2}{c}{ Temperature $\left({ }^{\circ} \mathrm{C}\right)$} \\
& For 50\% wt loss & For 65\% wt loss \\
\hline PK-CRG & 272.94 & 400.47 \\
K-CRGN0.5 & 286.89 & 421.15 \\
K-CRGN1.0 & 287.18 & 499.18 \\
K-CRGN1.5 & 304.20 & 532.09 \\
\hline
\end{tabular}

decomposition of the nanocomposites was studied for a wide range of compositions by systematically changing the nature of the macromolecule (polymer) or the nanofiller.

The NSI does not exhibit thermal degradation processes in the temperature interval investigated, so that the mass loss evidenced for the nanomaterials was ascribed to the polymer degradation. Figure 8 shows the thermal degradation curves obtained for K-CRG and K-CRG/NSI composite films. The thermal degradation of pure K-CRG film and K-CRG/NSI composite films curve exhibits relevant variations in the decomposition mechanism, as shown in Table 3. Thermal degradation temperature increased with increase in concentration of NSI in K-CRG. Temperature for 50\% degradation has increased from 272.94 to $304.20^{\circ} \mathrm{C}$, which is an increase of $11.5 \%$, on just $1.5 \%$ addition of NSI in K-CRG. Similarly, for $65 \%$ degradation, temperature has increased from 400.47 to $532.09^{\circ} \mathrm{C}$, which is an increase of $32.9 \%$. Thermally insulating nature of silica and the increase in crystallinity of K-CRG caused by addition of NSI (due to better compatibility) are the prime reasons for the degradation property observed. Thus, we can say that K-CRG/NSI composite films reflect an improvement in the resistance to the thermal degradation of the polymer, exercised by the NSI particles.

4.4. Barrier Properties. Figure 9 depicts the effect of NSI addition on the WVTR property of K-CRG. It was found that WVTR decreased for $0.5 \%$ addition of NSI in K-CRG and then started increasing for higher concentrations of NSI.

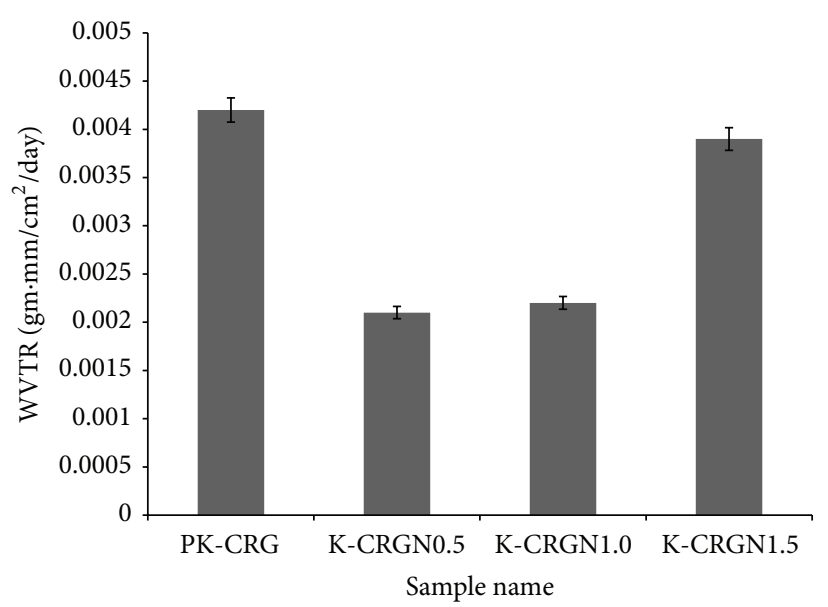

FIGURE 9: Effect of NSI concentration on WVTR of K-CRG films.

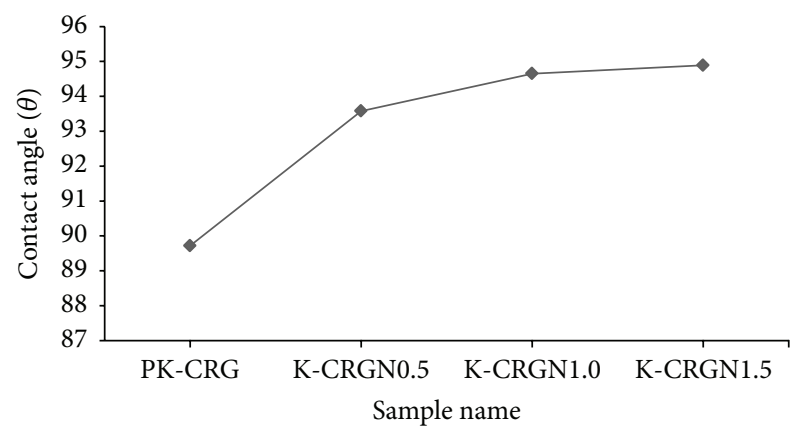

FIGURE 10: Effect of NSI concentration on the contact angle of KCRG films.

Increase in crystallinity caused due to better interaction between NSI and K-CRG (both being hydrophilic) decreased the intermolecular distance between K-CRG polymeric chains. This provided high resistance to the water molecules trying to cross over the films thickness, decreasing the WVTR. However, above $0.5 \%$ concentration, NSI formed aggregates, decreasing the effective surface area for interacting with NSI. This led to decrease in the crystallinity of the composite films, increasing the WVTR. WVTR was found to have decreased by $50 \%$ on just $0.5 \%$ addition of NSI. However, K-CRGN1.0 and K-CRGN1.5 still have lower WVTR than PK-CRG, due to higher crystallinity of them compared to PK-CRG. Also, NSI itself acted as water barrier material, decreasing the ability of water to go through the K-CRG film.

4.5. Contact Angle Measurement. Another interesting property of the composite film is the surface hydrophobicity and wettability, which was evaluated by means of contact angle determination. Contact angles of PK-CRG and KCRG/NSI composite films are illustrated in Figure 10. The results showed small increase in contact angle values of the nanocomposite films with NSI addition. This suggests an increase in film surface hydrophobicity due to the crystallinity increase caused by better interaction between NSI and K-CRG. These results indicate that the wettability of 

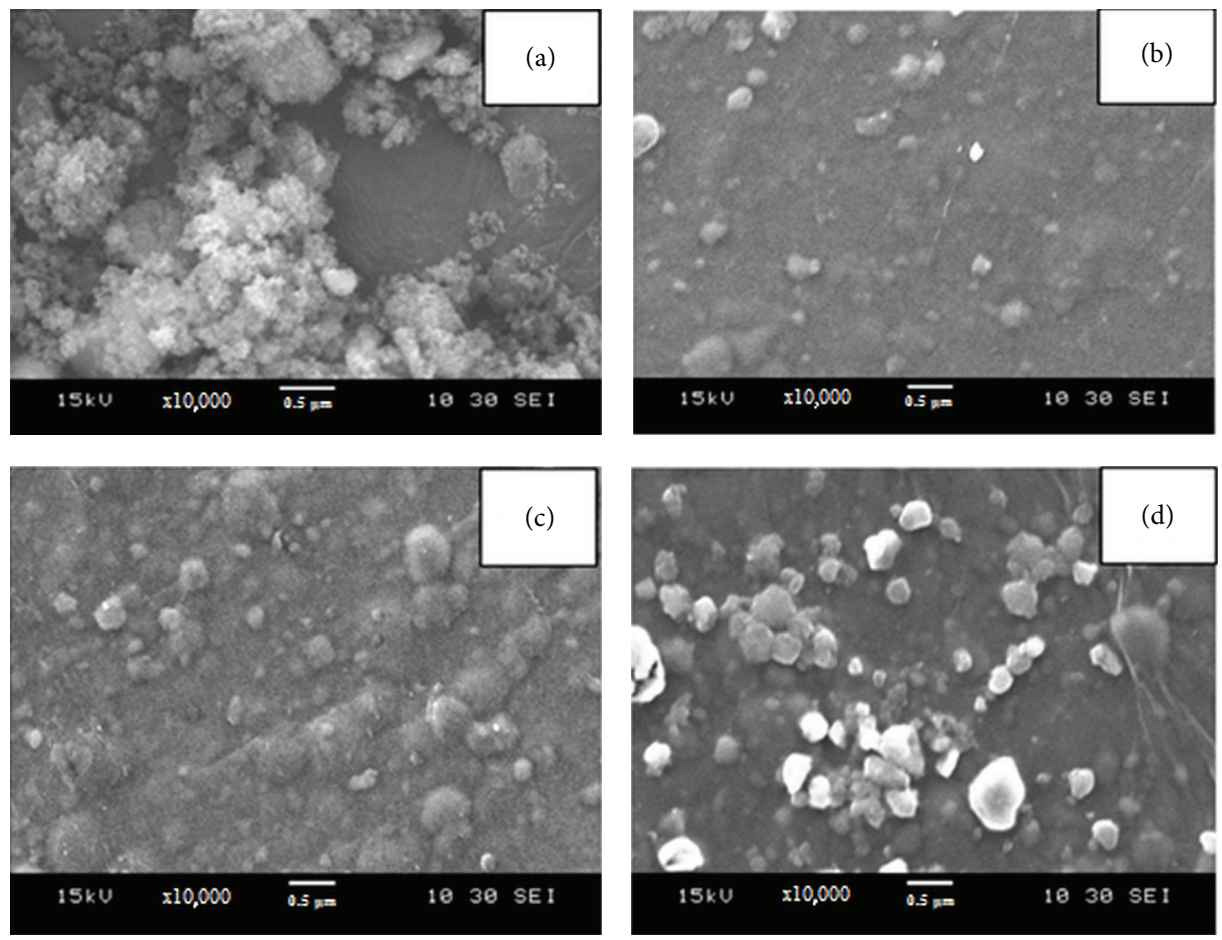

FIGURE 11: Scanning electron micrographs obtained for (a) PK-CRG, (b) K-CRGN0.5, (c) K-CRGN1.0, and (d) K-CRGN1.5.

the composite films decreased with an increase in the NSI content.

4.6. Morphological Analysis. The scanning electron microscopy (SEM) analysis of the nanocomposites films and modified nanosilica was done at $15 \mathrm{kV}$ power and $10 \mathrm{~K}$ magnification. SEM images obtained for PK-CRG and K-CRG/NSI composite films are shown in Figure 11(a-d).

Figure 11(a) shows the SEM image for NSI. NSI can be seen to be spherical particles with spherical shape. Figure 11(b), Figure 11(c), and Figure 11(d) show the SEM images obtained for K-CRGN0.5, K-CRGN1.0, and K-CRGN1.5, respectively. NSI was found to be uniformly dispersed in KCRGN0.5, whereas aggregates were found to have formed in K-CRGN1.0 and K-CRGN1.5. This is in connection with the trend observed in the mechanical, crystallinity, and WVTR properties obtained earlier.

4.7. Rheological Properties. K-CRG ( $1 \mathrm{wt} \%$ in solution in water) showed detectable shear thinning (reduction in viscosity with increasing shear rate), as is commonly observed [19] for solutions of entangled polymer coils. Figure 12 illustrates variation of shear viscosity $(\mathrm{Pa} \cdot \mathrm{s})$ at $30^{\circ} \mathrm{C}$ with NSI concentration for shear rates from 0.1 to $1000 \mathrm{~s}^{-1}$. Shear viscosity of K-CRGN0.5 is higher than PK-CRG at low shearing rates. However, K-CRGN1.0 and K-CRGN1.5 showed low shear viscosity as compared to PK-CRG. Figures 13 and 14 show the frequency dependence of the dynamic moduli $\left(G^{\prime}\right.$ and $\left.G^{\prime \prime}\right)$ for PK-CRG and K-CRG/NSI composites. Storage modulus $\left(G^{\prime}\right)$ increased for $0.5 \%$ concentration of NSI but then started decreasing for higher concentrations of NSI.

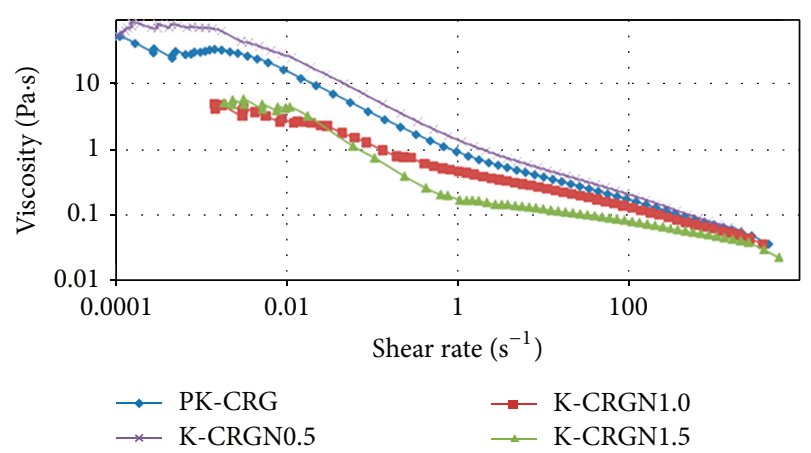

FIGURE 12: Effect of NSI concentration on viscosity of K-CRG (1\% solution in water).

The increase in storage modulus verifies increase in mechanical properties. Loss modulus $\left(G^{\prime \prime}\right)$ also increased for KCRGN0.5 compared to PK-CRG. But higher percentage addition of NSI showed the lower loss modulus compared to PK-CRG. Figure 15 shows the variation of $\tan \delta$ with frequency for all the nanocomposites of K-CRG/NSI at $30^{\circ} \mathrm{C}$. It is very clear from this figure that $\tan \delta$ values are lowered when NSI is added to the K-CRG.

Rheological properties show that the interfacial adhesion between K-CRG and NSI was higher for K-CRGN0.5, but then after K-CRGN1.0 and K-CRGN1.5 there is a drastic decrease, which means that $0.5 \%$ is threshold composition value. The NSI seems to be responsible for the improved rheological properties, which is proof for the observed improvement in the mechanical properties. 


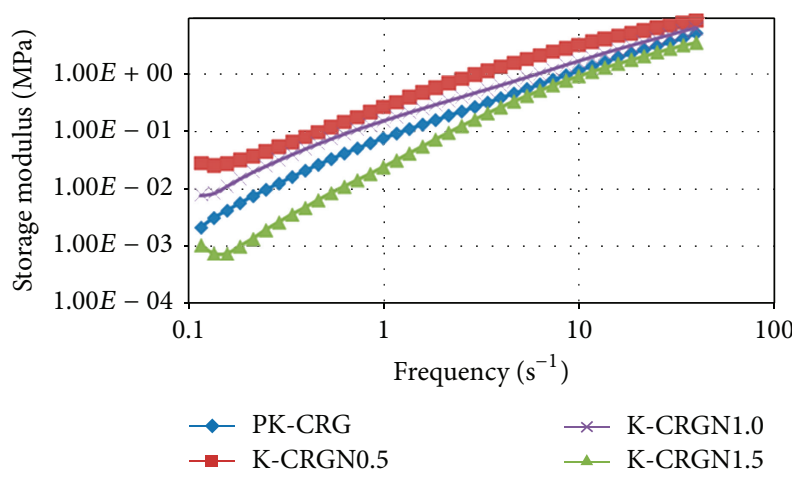

FIGURE 13: Effect of NSI concentration on storage modulus of KCRG ( $1 \%$ solution in water).

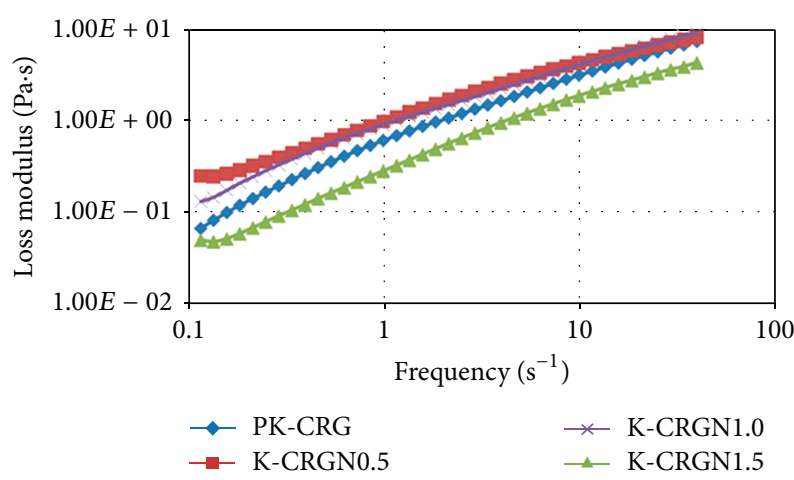

FIGURE 14: Effect of NSI concentration on loss modulus of K-CRG ( $1 \%$ solution in water).

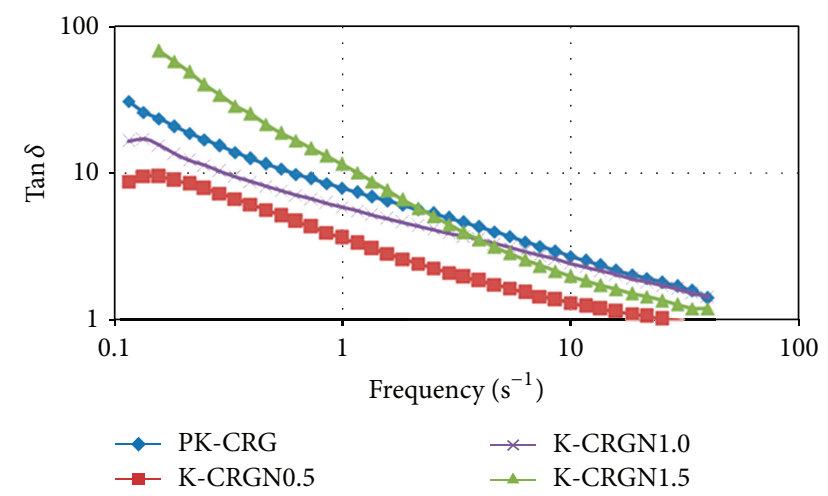

FIGURE 15: Effect of NSI concentration on the phase angle $(\tan \delta)$ of K-CRG ( $1 \%$ solution in water).

\section{Conclusions}

The composite films of K-CRG with various NSI concentrations were prepared by using solution casting method. DSC and TGA results of the composite films showed improved melting temperature, enthalpy, and thermal degradation temperatures of K-CRG biopolymer matrix on addition of NSI. The SEM results indicate formation of NSI agglomerates above $0.5 \%$ concentration in K-CRG biopolymer matrix. Tensile properties for composite films are dependent on
NSI dispersion and formation of composite structure. The investigation of water vapor penetration and wettability of the composite films is done by water vapour transmission rate (WVTR) and contact angle, respectively. The composition of these composite films and its resulting structure are thus thought to affect their water barrier properties as well as wettability of the K-CRG biopolymer matrix films. Rheological properties showed good improvement in viscosity and storage modulus for $0.5 \%$ NSI added K-CRG samples. This knowledge of the composite film could make beneficial contributions to the food and pharmaceutical packaging applications.

\section{Conflict of Interests}

The authors declare that there is no conflict of interests regarding the publication of this paper.

\section{References}

[1] A. K. Mohanty, L. T. Drzal, and M. Misra, "Nano reinforcements of bio-based polymers: the hope and the reality," Polymeric Materials Science and Engineering, vol. 88, pp. 60-61, 2003.

[2] F. Van de Velde and G. A. De Ruiter, Carrageenan Polysaccharides and Polyamides in the Food Industry, Wiley-VCH, Weinheim, Germany, 2005.

[3] C. L. Wu, M. Q. Zhang, M. Z. Rong, and K. Friedrich, "Tensile performance improvement of low nanoparticles filledpolypropylene composites," Composites Science and Technology, vol. 62, no. 10-11, pp. 1327-1340, 2002.

[4] M. W. Lee, X. Hu, L. Li, C. Y. Yue, K. C. Tam, and L. Y. Cheong, "PP/LCP composites: effects of shear flow, extensional flow and nanofillers," Composites Science and Technology, vol. 63, no. 13, pp. 1921-1929, 2003.

[5] M. M. Hasan, Y. Zhou, H. Mahfuz, and S. Jeelani, "Effect of $\mathrm{SiO}_{2}$ nanoparticle on thermal and tensile behavior of nylon-6," Materials Science and Engineering A, vol. 429, no. 1-2, pp. 181$188,2006$.

[6] A. Sargsyan, A. Tonoyan, S. Davtyan, and C. Schick, "The amount of immobilized polymer in PMMA SiO2 nanocomposites determined from calorimetric data," European Polymer Journal, vol. 43, no. 8, pp. 3113-3127, 2007.

[7] R. D. Priestley, P. Rittigstein, L. J. Broadbelt, K. Fukao, and J. M. Torkelson, "Evidence for the molecular-scale origin of the suppression of physical ageing in confined polymer: fluorescence and dielectric spectroscopy studies of polymer-silica nanocomposites," Journal of Physics Condensed Matter, vol. 19, no. 20, Article ID 205120, 2007.

[8] K. Chrissafis, K. M. Paraskevopoulos, E. Pavlidou, and D. Bikiaris, "Thermal degradation mechanism of HDPE nanocomposites containing fumed silica nanoparticles," Thermochimica Acta, vol. 485, no. 1-2, pp. 65-71, 2009.

[9] E. F. Voronin, V. M. Gun'ko, N. V. Guzenko et al., "Interaction of poly(ethylene oxide) with fumed silica," Journal of Colloid and Interface Science, vol. 279, no. 2, pp. 326-340, 2004.

[10] K. Chrissafis, K. M. Paraskevopoulos, G. Z. Papageorgiou, and D. N. Bikiaris, "Thermal and dynamic mechanical behavior of bionanocomposites: fumed silica nanoparticles dispersed in poly(vinyl pyrrolidone), chitosan, and poly(vinyl alcohol)," Journal of Applied Polymer Science, vol. 110, no. 3, pp. 1739-1749, 2008. 
[11] J. Lee, K. Jin Lee, and J. Jang, "Effect of silica nanofillers on isothermal crystallization of poly(vinyl alcohol): in-situ ATRFTIR study," Polymer Testing, vol. 27, no. 3, pp. 360-367, 2008.

[12] Y.-L. Chung, S. Ansari, L. Estevez, S. Hayrapetyan, E. P. Giannelis, and H.-M. Lai, "Preparation and properties of biodegradable starch-clay nanocomposites," Carbohydrate Polymers, vol. 79, no. 2, pp. 391-396, 2010.

[13] N. R. Savadekar, V. S. Karande, N. Vigneshwaran, A. K. Bharimalla, and S. T. Mhaske, "Preparation of nano cellulose fibers and its application in kappa-carrageenan based film," International Journal of Biological Macromolecules, vol. 51, pp. 10081013, 2012.

[14] N. R. Savadekar and S. T. Mhaske, "Synthesis of nano cellulose fibers and effect on thermoplastics starch based films," Carbohydrate Polymers, vol. 89, no. 1, pp. 146-151, 2012.

[15] S. Jose, S. Thomas, P. K. Biju, P. Koshy, and J. Karger-Kocsis, "Thermal degradation and crystallisation studies of reactively compatibilised polymer blends," Polymer Degradation and Stability, vol. 93, no. 6, pp. 1176-1187, 2008.

[16] V. Rao and J. Johns, "Thermal behavior of chitosan/natural rubber latex blends TG and DSC analysis," Journal of Thermal Analysis and Calorimetry, vol. 92, no. 3, pp. 801-806, 2008.

[17] D.-W. Lee, S.-J. Park, S.-K. Ihm, and K.-H. Lee, “One-pot synthesis of Pt-nanoparticle-embedded mesoporous titania/silica and its remarkable thermal stability," Journal of Physical Chemistry C, vol. 111, no. 21, pp. 7634-7638, 2007.

[18] X. Yuan, C. Li, G. Guan, Y. Xiao, and D. Zhang, "Thermal degradation investigation of poly(ethylene terephthalate)/fibrous silicate nanocomposites," Polymer Degradation and Stability, vol. 93, no. 2, pp. 466-475, 2008.

[19] J. P. Busnel and S. B. Rose-Murphy, "Thixotropic behaviour of very dilute gelatin solutions," International Journal of Biological Macromolecules, vol. 10, no. 2, pp. 121-124, 1988. 

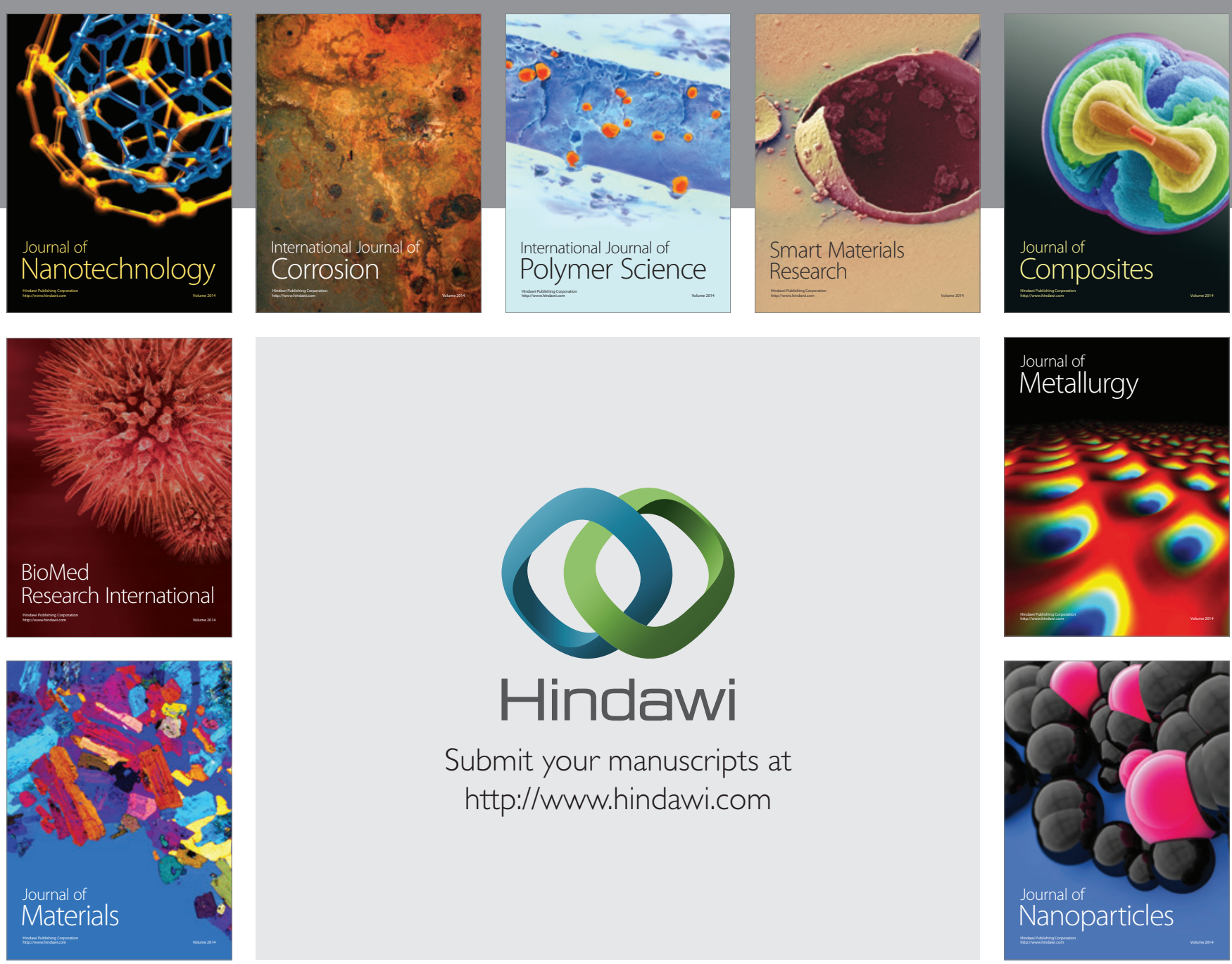

Submit your manuscripts at http://www.hindawi.com
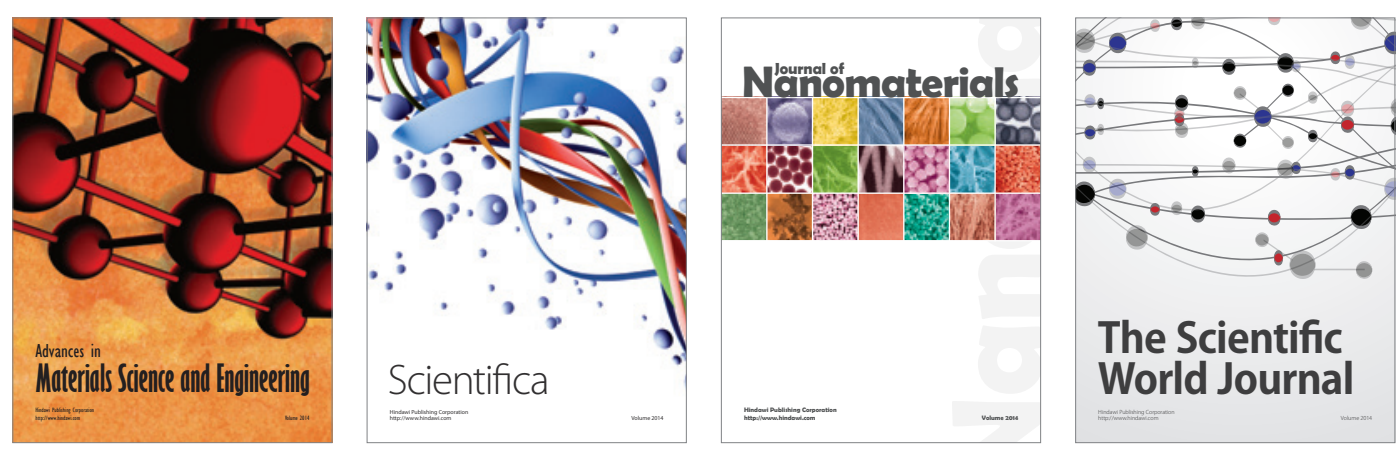

\section{The Scientific World Journal}
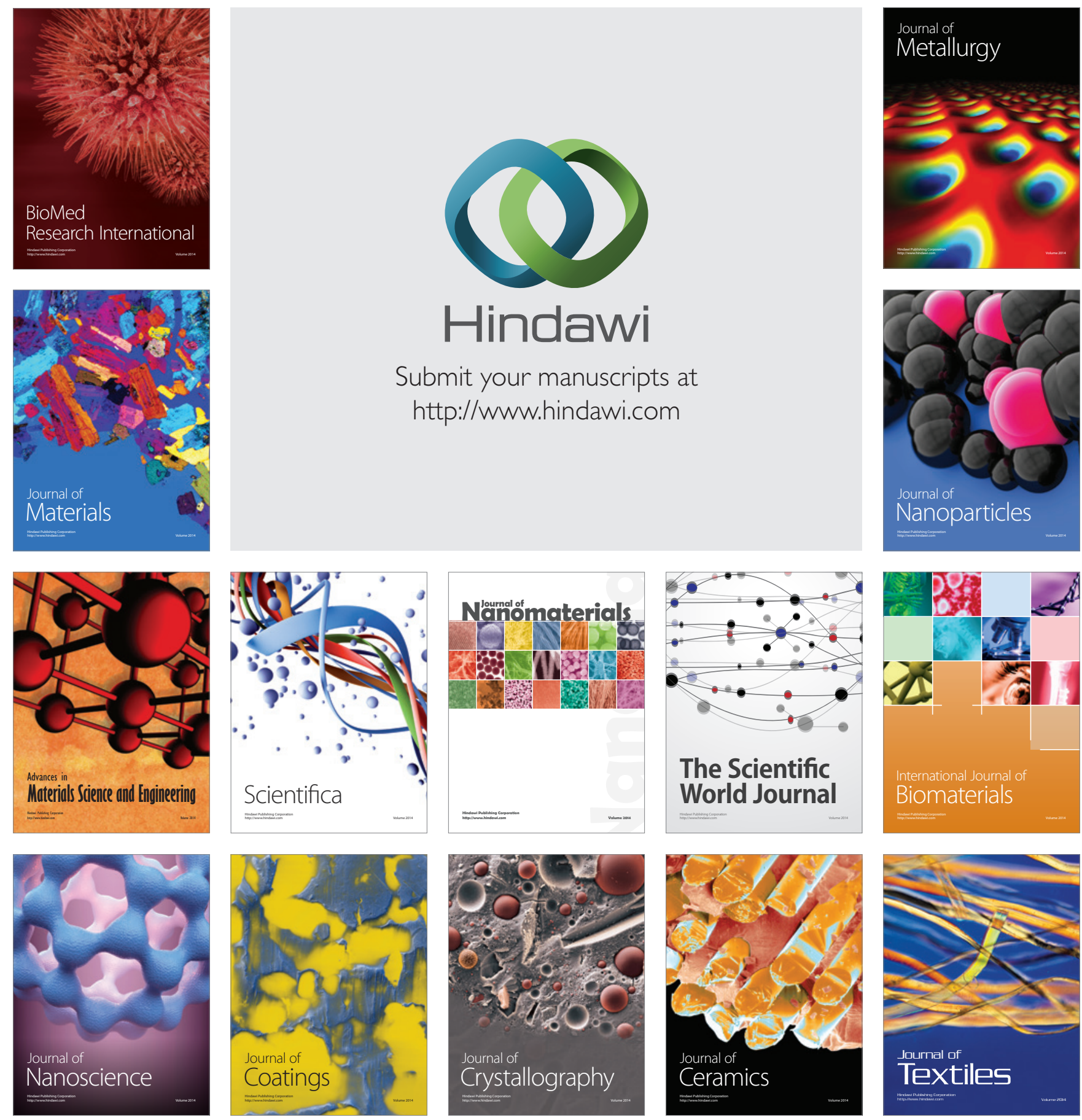